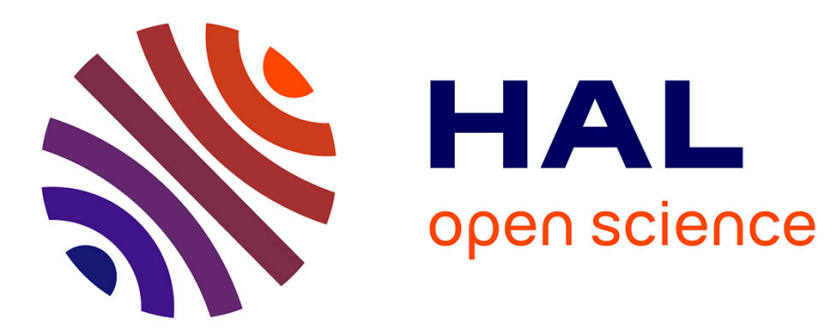

\title{
L'impossible réparation : l'image brisée de François Fillon durant la campagne présidentielle française 2017
}

Magali Guaresi, Damon Mayaffre

\section{To cite this version:}

Magali Guaresi, Damon Mayaffre. L'impossible réparation: l'image brisée de François Fillon durant la campagne présidentielle française 2017. Langage et Société, 2018. hal-01870751

\section{HAL Id: hal-01870751 \\ https://hal.science/hal-01870751}

Submitted on 9 Sep 2018

HAL is a multi-disciplinary open access archive for the deposit and dissemination of scientific research documents, whether they are published or not. The documents may come from teaching and research institutions in France or abroad, or from public or private research centers.
L'archive ouverte pluridisciplinaire HAL, est destinée au dépôt et à la diffusion de documents scientifiques de niveau recherche, publiés ou non, émanant des établissements d'enseignement et de recherche français ou étrangers, des laboratoires publics ou privés. 
"L'impossible réparation : l'image brisée de François Fillon durant la campagne présidentielle française 2017", Langage et Société, 164, 2018, pp. 39-56.

Magali Guaresi et Damon Mayaffre (UMR 7320 Bases, Corpus, Langage Université Côte d'Azur)

Magali.GUARESI@unice.fr

DamonMayaffre@,wanadoo.fr

\section{L'impossible réparation: l'image brisée de François Fillon durant la campagne présidentielle française 2017}

Cette contribution pose la stratégie de restauration ou de réparation d'image, telle que théorisée par William Benoit, comme un acte de langage. Dès lors, elle s'interroge sur les conditions de félicité ou d'infélicité de ce speech act. Durant la campagne présidentielle française 2017, le candidat favori, François Fillon, a vu son image d'homme sincère se briser sur les affaires sans être capable, malgré les efforts déployés, de la réparer. En utilisant successivement et/mais de manière contradictoire la 'mortification', la 'minimization'-, le 'denial', la 'compensation', l'attacking accuser', la 'provocation', l'evading responsibility' François Fillon a semblé affaiblir sa défense et rendre irrecevable son discours. De même, en utilisant parfois le logos, parfois le pathos, parfois l'ethos, il semble avoir perdu la cohérence rhétorique nécessaire à l'efficacité. Enfin, cette contribution pointe l'aporie de toute stratégie discursive de restauration lorsque c'est la parole du locuteur qui se trouve elle-même discréditée : comment réparer par le discours, un discours devenu inaudible?

Mots clefs: restauration d'image, présidentielle 2017, François Fillon, discours politique, affaires

Abstract :

Keywords: image restoration, 2017 presidential campaign, François Fillon, political discourse, political scandal 


\section{Introduction}

A posteriori, c'est-à-dire à quelques mois de distance des événements, il est possible de constater que la tentative de réparation d'image que cette contribution va essayer de décrire s'est soldée par un échec. De fait, au terme du processus, le protagoniste - François Fillon, ancien Premier ministre de la France et candidat à la présidentielle 2017 - a dû abandonner la vie politique après avoir été incapable de se qualifier au second tour de l'élection majeure française : sa carrière politique tout entière est aujourd'hui brisée après que son image a été irréparablement cassée.

Dès lors, l'enjeu de ces lignes n'est pas de supposer que François Fillon, pris dans une tempête médiatico-judiciaire qui mettait à bas sa crédibilité personnelle et son éthos d'honnête homme, n'a rien entrepris pour restaurer son image mais de comprendre, au contraire, comment le candidat a entrepris trop et trop mal pour que sa stratégie de restauration s'avère gagnante : ce sont les conditions de félicité - ou en l'occurrence les condition d'infélicité - de la restauration d'image qui seront ainsi interrogées. Et nous reprenons ici à dessein la notion de felicity conditions car nous posons la réparation d'image comme un acte de discours par excellence, à juger non pas en termes de véri-conditionnalité mais en termes de satisfaction/insatisfaction [Austin 1962 ; Searle 1969].

Pendant trois mois en effet, comme nous le verrons, François Fillon a usé sans relâche, successivement ou simultanément, de toutes les stratégies rhétoriques possibles pour réparer son image. Les stratégies que pointent William Benoit [Benoit 1995] et ses collaborateurs bien sûr ${ }^{1}$, et d'autres stratégies encore : le déni puis la dilution des responsabilités, la minimisation de l'offense et de ses conséquences pour son image, l'action corrective - François Fillon a déclaré par exemple « avoir rendu » les costumes dont il avait hérité gratuitement - et le mea culpa; surtout, au fil de la campagne, il a usé du retournement de la charge. De coupable, François Fillon s'est présenté en victime des médias, de la justice ou du cabinet noir de François Hollande, et d'accusé il est devenu alors accusateur ; c'est par la contre-attaque qu'il a tenté aussi de restaurer son image.

Seulement, à force de se multiplier, les arguments se sont annulés loin de se cumuler. Le postulat est que la multiplication de productions argumentatives devient contre-productive ; à trop dire, on risque de se contredire et l'image du locuteur en sort plus brouillée encore. Par exemple, il est difficile de nier la faute en même temps que de s'excuser de l'avoir commise ; et contre-attaquer violemment n'est-ce pas d'une certaine manière donner crédit à l'attaque ? User du pathos ici, lorsque le candidat déclarait en tribune aimer et vouloir protéger sa femme, n'est-ce pas condamner le logos utilisé là, lorsqu'il affirmait vouloir

\footnotetext{
${ }^{1}$ Nous reprendrons ainsi dans cet article les concepts-clefs ou catégories argumentatives de Benoit et de son école, et les notons par convention en italique et entre apostrophe :'mortification', 'minimization', 'denial', 'compensation', 'attacking accuser', 'provocation', 'evading responsibility'. Les 6 références bibliographiques majeures utilisées, dont [Benoit 1995], sont notées en fin d'article.
} 
seulement penser rationnellement au pays, pour un éthos au final rendu illisible ? En d'autres termes, la félicité de la restauration d'image passe par une cohérence du discours que François Fillon semble n'avoir pas été en mesure de respecter face à la pression du calendrier, des médias, des juges et de l'opinion publique.

\section{Contexte}

Le 14 mars 2017, en pleine campagne électorale et à quelques semaines du scrutin, le candidat favori de l'élection présidentielle française, François Fillon, est mis en examen notamment pour détournement de fonds publics, complicité et recel de détournement de fonds publics, complicité et recel d'abus de bien sociaux. Aux accusations de la presse qui faisait état depuis 20 jours, et l'article du Canard enchainé du 25 janvier, de l'emploi présumé fictif de sa femme, durant une dizaine d'années, pour une somme proche d'1 million d'euros s'ajoutait ainsi un acte judiciaire officiel qui devait écrouler définitivement l'image du candidat.

Les "affaires Fillon ", que l'on peut mettre au pluriel tant les révélations de faits avérés comme inventés se sont multipliées dès lors dans les journaux ${ }^{2}$, ont été d'autant plus mortifères pour le candidat que François Fillon cultivait jusqu'ici un ethos d'homme intègre voire austère, et qu'il avait lui-même construit sa campagne électorale, notamment pendant la Primaire de la droite et du centre en 2016, sur le double discours de l'exemplarité morale nécessaire des dirigeants politiques et des efforts d'ascèse, de travail et d'économie indispensables pour les Français et le pays.

Particulièrement, en rivalité dans son propre parti avec Nicolas Sarkozy, en butte lui-même avec la justice française, François Fillon avait déclaré pour disqualifier son concurrent : « Qui imagine le général de Gaulle mis en examen ? " (F. Fillon, 28 août 2016, discours dans la Sarthe). Et au départ de l'affaire, niant encore tout en bloc et criant au complot médiatique, François Fillon avait concédé au journal télévisé de TF1 : " I n'y a qu'une seule chose qui m'empêcherait d'être candidat c'est si mon honneur était atteint, si j'étais mis en examen... " (F. Fillon, 26 janvier 2017, interview télévisée). ${ }^{3}$

\footnotetext{
${ }^{2}$ Nous ne retracerons pas ici la chronique et n'essayerons pas de démêler le faux du vrai d'autant que des procédures judiciaires sont encore en cours. Mais outre l'emploi présumé fictif de sa femme et de ses enfants, des soupçons de corruption ont été avancés après que l'on eut découvert que l'homme politique se faisait offrir des costumes de luxe par un avocat d'affaire, Robert Bourgi, lié à la Françafrique. Se sont ajoutés dès lors d'autres soupçons moins étayés sur l'absence de déclaration fiscale de certains crédits du candidat ou de liens sulfureux avec la Russie via la société de conseil de François Fillon « F2 Conseil », etc.

${ }^{3}$ Lors de sa conférence de presse du 6 février 2017, François Fillon réédite, plus clairement encore, son propos : " J'ai dit que mis en examen, je ne serai(s) pas candidat à l'élection présidentielle [...] Je pense être le seul candidat à l'élection présidentielle [...] à avoir pris un tel engagement. [...] (La situation actuelle)... ne change rien à l'engagement que j'ai pris ".
} 
En d'autres mots, au soupçon de corruption et d'emploi fictif pour sa famille, s'ajoutait dès lors qu'il était mis en examen celui de parjure. Homme d'argent d'un côté et de peu de parole de l'autre : deux des plus graves accusations en politique frappaient le candidat, non sans quelques éléments vérifiables (luxueux manoir de famille pris en photo par la presse, extraits d'interview de sa femme démentant avoir travaillé ou de lui-même passés en boucle à la radio ou à la télévision ${ }^{4}$ ): devant une situation devenue intenable, les conseils d'abandon venant de ses adversaires comme de son propre camp politique se multiplièrent ; et l'on envisagea de remplacer le malheureux candidat par son dauphin de la Primaire, Alain Juppé ou par un jeune homme neuf tel François Baroin. Pourtant, François Fillon a maintenu sa candidature jusqu'au bout en espérant restaurer son image et devenir président.

\section{L'excuse dément le déni}

Le 6 février 2017, deux semaines après les révélations du Canard enchainé auxquelles l'ensemble de la presse française et internationale donnait écho, François Fillon se trouve contraint de convoquer les journalistes pour justifier la rémunération de sa femme et de ses enfants, et clore, espère-t-il, l'épisode qui abîme son image. A son QG de campagne, au pupitre et devant les caméras de télévision, il déclare :

(1) C'était une erreur. Je le regrette profondément. Et je présente mes excuses aux Français. (F. Fillon, 6 février 2017, conférence de presse)

Quelques jours après, lors d'une grande manifestation de soutien organisée par ses partisans à Paris au Trocadéro, il précise à la tribune :

(2) Je vous dois des excuses, dont celles de devoir défendre mon honneur et celui de mon épouse, alors que l'essentiel est, pour vous comme pour moi, de défendre notre pays. [...]. J'ai commis la première erreur, je vous l'ai dit, en demandant à mon épouse de travailler pour moi parce qu'elle connaissait le terrain, parce que c'était commode. Je n'aurais pas dû le faire. Et j'ai commis la seconde en hésitant sur la manière d'en parler, de vous en parler, d'en parler aux Français. (F. Fillon, 5 mars 2017, meeting de Paris)

\footnotetext{
${ }^{4}$ Outre le « Qui imagine le général de Gaulle mis en examen ? " qui visait Nicolas Sarkozy, l'autre extrait, qui visait Alain Juppé son concurrent du soir lors du débat télévisé de La Primaire, et ancien condamné de Justice, a été ainsi beaucoup rappelé : «On ne peut pas diriger la France si on n'est pas irréprochable. [...] J'ai déjà eu l'occasion de le dire, ça a créé des émois. C'est une évidence. Les ministres et le président de la République ne doivent pas être mis en examen, et doivent avoir une attitude exemplaire. [...] Je sais que ça n'est pas juste, qu'il y a la présomption d'innocence, mais l'expérience qui est la mienne montre qu'on ne peut pas diriger sereinement un ministère si on a une suspicion sur le dos. Les hommes politiques ne sont pas citoyens comme les autres. Ils doivent montrer le chemin... " (F. Fillon, 24 novembre 2016, débat télévisé de l'entre-deux tours de la Primaire de droite et du centre).
} 
Par deux fois, le substantif "excuse" au pluriel et selon les tournures habituelles (" présenter ses excuses..., devoir des excuses... ») se trouve donc utilisé par le candidat. Dans la typologie de William Benoit [Benoit 1995], François Fillon utilise ainsi la 'mortification' comme stratégie de restauration. Le " je le regrette profondément " de l'extrait 1 confirme la posture expiatoire qui nous mène sur les rives du pardon demandé ou de la contrition.

Seulement, les excuses sont accompagnées dans le discours d'une minimalisation ('minimization') voire d'une négation ('denial') du préjudice qui nous parait empêcher le performatif (s'excuser) d'être performant.

Sans revenir sur les déclarations initiales puis éparses du candidat qui montrent que François Fillon commence par nier les faits ${ }^{5}$, on note dans les deux extraits que François Fillon refuse non seulement l'idée de délit mais aussi de faute pour parler seulement, dans un choix lexical contresigné " d'erreur ». Certes, à strictement parler, s'excuser pour une erreur n'est pas impropre, mais l'erreur semble ne relever que de la technique lorsque l'excuse mobilise l'éthique. L'erreur est involontaire, accidentelle, contingente, lorsque la faute - si François Fillon avait utilisé ce mot - aurait renvoyé à la responsabilité sinon à la culpabilité de son auteur. En un mot, François Fillon s'excuse pour une faute qu'il dit ne pas avoir commise et se trouve entrainé dans une procédure judiciaire pour un délit qu'il dit ne pas avoir perpétré $^{6}$ : loin de réparer son image, François Fillon apparaît comme contradictoire et insincère dans sa demande de pardon en raison des ambiguïtés ('minimization' concomitante) que sa posture et sa communication comportent.

La presse immédiatement s'engouffre dans ce presque dit et ce faux mea culpa. Le quotidien La Croix par exemple glose dans un article intitulé "Fillon, la faute et l'erreur» sur la " distinction entre ce qui est légal, éthique et politique » (B. Bouniol, La Croix, 07 février 2017) et convoque pour cela la casuistique et la philosophie. Il en ressort globalement que si l'on doit certes corriger ses erreurs, il n'y a pas lieu d'en demander pardon ou bien de s'en excuser. Et un conseiller en communication de réclamer à la radio, en préalable à toute excuse, que le candidat reconnaisse clairement la "faute morale » qu'il a commise (P. Moreau Chevrolet, $R T L, 01 / 02 / 2017)$. Sans cela, pourrait-on dire en paraphrasant Benoit et Brinson [1994] qui affirment que parfois l'excuse n'est pas suffisante, que l'excuse n'est pas pertinente.

\footnotetext{
${ }^{5}$ Les dénégations de François Fillon sont complexes et pourraient être décrites dans le détail. François Fillon n'a jamais nié avoir embauché sa femme et ses enfants, mais il nie, encore à ce jour, que leur travail ait été fictif. II conteste également les dates et périodes qui ont pu être avancées ou la hauteur des rémunérations que la presse a divulguée. Plus fermement, il nie avoir été en affaire avec la Russie ou les trafics d'influence dont il est soupçonné via sa société " $2 \mathrm{~F}$ Conseil ». Il réfute également être intervenu auprès de son ami Marc Ladreit de Lacharrière pour l'embauche de sa femme à la Société des deux mondes.

${ }^{6}$ Dans le détail, l'extrait 2 parait caricatural : François Fillon dit s'excuser, mais s'excuse avant tout d'être obligé de défendre son honneur et celui de sa femme ; non pas d'avoir commis un forfait.
} 
Les électeurs, eux en tout cas, semblent ne pas pouvoir valider l'acte de contrition : si faute avouée est à moitié pardonnée, faute qu'à moitié avouée reste impardonnée. L'infélicité de l'acte de langage (s'excuser) est patente: le locuteur présente des excuses que l'auditeur ne saurait accepter puisqu'elles portent sur des faits minimisés sinon déniés. Pire : en s'excusant, François Fillon atteste, comme malgré lui, de l'existence d'une faute qu'il s'obstine de manière contradictoire à contester. L'excuse est non reçue; la faute est confirmée ; et l'image irréparée.

\section{La réparation ('compensation') confirme le délit}

(3) Un ami m'a offert des costumes en février. Et alors ? (F. Fillon, 12 mars 2017, interview aux Echos)

(4) Je les ai rendus (F. Fillon, 23 mars 2017, interview télévisée)

$C^{\prime}$ est en peu de mots que François Fillon pense tourner la page de l'affaire des costumes ${ }^{7}$ et restaurer son image : 'minimization' d'abord, 'compensation' ensuite.

Ici à nouveau, les deux stratégies théorisées par la science de la communication se contredisent pour brouiller sinon dégrader un peu plus l'image du candidat. Si bénéficier d'un cadeau était banal et n'avait rien de répréhensible ('minimization') comme semble l'indiquer la formulation populaire de la question rhétorique "et alors ? " (extrait 3), pourquoi François Fillon a-t-il eu finalement besoin de restituer ledit cadeau ('compensation') et de faire publicité de cette restitution (extrait 4) y compris devant les juges?

Enfermé dans cette contradiction élémentaire, François Fillon ne sortira pas de son cercle carré, et la presse appuiera des deux côtés où l'argumentation blesse.

(i) La restitution des costumes réparait-elle le préjudice ('compensation') et lavait-elle ainsi le candidat de la faute passée ? Et les journaux d'ironiser alors sur la réutilisation par le quidam de costumes de luxe d'occasion taillés sur mesure pour le candidat, ou sur le fait que François Fillon se serait trompé dans les modèles rendus : si François Fillon pensait restaurer son image d'homme intègre, c'était en construisant l'image du riche gaspilleur, sans conscience de la valeur des choses, qui seyait mal au programme d'économie et d'effort qu'il présentait par ailleurs.

\footnotetext{
${ }^{7}$ Affaire dans l'affaire, le Journal du dimanche publie le 11 mars 2017 un article révélant que François Fillon s'était fait offrir des costumes de luxe par d'anonymes donateurs pour une somme totale de 48.500 euros depuis 2012. Cette affaire des costumes sera rapidement versée à l'enquête principale sur les emplois présumés fictifs de la famille Fillon, et les chefs d'inculpation initiaux du candidat furent élargis à « trafic d'influence ». Plusieurs interviews télé et radio du principal protagoniste - Robert Bourgi - ont été diffusées durant l'hiver 2017-2018 qui éclairent d'une sombre lumière les événements.
} 
(ii) Les cadeaux eux-mêmes étaient-ils naturels et irréprochables ('minimization') ? Et la presse de préciser ici que le règlement de l'Assemblée nationale rend obligatoire depuis 2011 la déclaration de tout don ou avantage d'une valeur supérieure à 150 euros, là où les costumes offerts auraient coûté depuis 2012 près de 50.000 euros $^{8}$; dans ce cas, c'était bien I'intégrité de François Fillon et son manque de transparence qui se trouvaient discutés, là où le candidat s'était présenté comme un père la vertu, un homme de rigueur irréprochable ou comme « le candidat de la vérité » (F. Fillon, 3 janvier 2017, interview télévisée).

Et c'est au niveau discursif que la séquence est intéressante. Si l'on pose la réparation d'image comme un acte de langage qui doit performer un éthos réhabilité, sa première condition de réalisation tient dans la cohérence interne au discours qui ne peut fabriquer ou performer en même temps l'image de l'innocent (" et alors ? ») et celui du repenti (" je les ai rendus "): si l'on peut peut-être dire le tout et son contraire, il est en revanche difficile de faire (performer) les opposés.

\section{La contre-attaque valide l'attaque}

(5) Un livre qui porte sur le Ministère de I'Intérieur et la place Beauvau, qui en 240 pages explique comment François Hollande fait remonter toutes les écoutes judiciaires qui l'intéressent à son bureau. (F. Fillon, 23 mars 2017, interview télévisée, France 2)

(6) Le journaliste : C'est Hollande qui a déclenché l'affaire ?

François Fillon : Oui, bien sûr. (F. Fillon, 6 avril 2017, interview radio, France Inter)

François Fillon utilise ici l'attacking accuser' pour retourner les charges qui pèsent sur lui et passer d'inculpé à victime, d'accusé à accusateur. II avance ainsi plusieurs fois durant la campagne l'idée d'un cabinet noir à l'Elysée dirigé contre lui par François Hollande; ce que le président de la République évidemment mais aussi l'auteur de l'ouvrage convoqué dans l'extrait 5 démentiront fermement.

La stratégie aurait pu porter si François Hollande avait été un rival. Mais le Président affaibli n'était pas candidat et s'était mis en retrait de la compétition électorale, rendant ainsi illisible le mobile de la machinerie que François Fillon tente d'accréditer. Selon une stratégie que Benoit \& Hanczor [1994] et Benoit [1997] décrivent pour les affaires Tonya Harding et Hugh Grant, François Fillon cherche donc à se victimiser. Mais sans preuve, sans mobile, sans

\footnotetext{
${ }^{8}$ Nous n'entrerons pas dans le détail des maladresses de François Fillon. En effet, ici, sa première défense a été alors d'affirmer qu'il ne s'agissait pas d'un cadeau "politique " (donc soumis au règlement de l'Assemblée) mais d'un cadeau purement « amical »... de la part d'un ami à la réputation sulfureuse qui dénonça très vite et avec fracas cette amitié supposée dans la presse.
} 
adversaire explicite, sa contre-attaque porte à faux ; sa défausse apparaît fausse, dégradant ici encore un peu plus l'image d'un candidat insincère.

Plus problématique et plus contradictoire, poussant jusqu'au bout sa stratégie contreagressive, François Fillon s'en prend à la justice qu'il avait lui-même pourtant appelée en renfort. Réclamant d'abord d'être entendu par la justice et par le Parquet, il soupçonne ensuite le manque d'indépendance des juges (cf. infra) et accuse une enquête menée à charge. Accuser la justice d'injustice reste une stratégie risquée devant une opinion publique réservée face au monde politique, surtout lorsque l'on entend devenir le Président du pays c'est-à-dire le gardien des institutions.

Loin de passer pour une victime, François Fillon passera vite ainsi pour paranoïaque et tenant de la théorie du complot. Un cabinet noir politique, un procès en sorcellerie médiatique : des forces occultes se liguaient contre lui. Immédiatement, une presse sévère pouvait l'accuser de dresser un " rideau de fumée ${ }^{9}$ pour cacher sa culpabilité. A la radio, il menace pourtant :

(7) Et j'ai toutes les indications qui me permettront le moment venu de poursuivre ceux qui ont fait ça. Parce qu'il ne faut pas croire que je vais lâcher les accusations que j'ai lancées contre ceux qui ont déclenché cette opération... J'ai les dates, les jours, les personnes qui ont communiqué les documents, etc. ça viendra, le moment venu je poursuivrai tous ceux qui sont à l'origine de cette affaire. (F. Fillon, 6 avril 2017, interview radio, France Inter)

Techniquement, la performance, sinon la stricte performativité de l'acte de langage, on le sait, tient dans le présent de l'indicatif factif. Le "permettront le moment venu ", le " ça viendra ", le "je poursuivrai » de l'extrait 7 entraîneront ainsi des réactions négatives en chaîne, qui montrent l'infélicité de la réparation par la contre-attaque lorsqu'elle est conjuguée au futur. Entre mille exemples, le forum du site Riposte Laïque recueille beaucoup de réactions comme celles-ci où l'acte de langage a été ressenti comme un langage sans acte :

(8) Fillon ! CHICHE ! il ne suffit pas de brailler, montrer ses petits muscles; tu as des preuves ? sors-les, renverses la table, pour montrer que tu en as. Je crois plutôt, que tu n'as rien en magasin, tu fais du vent et que tu t'aplatiras comme une crêpe. Tu ne porteras

\footnotetext{
${ }^{9}$ C'est évidemment le soupçon et l'expression qui sont ressortis immédiatement. En français, dans la bouche d’une adversaire politique, Eva Joly: "Le rideau de fumée qu'essaye de créer François Fillon est insupportable » (Marianne, 18 février 2017). En anglais, sous la plume d’un journaliste du Telegraph : “Francois Fillon has just concluded the press conference of his political life [...]. It was an excellent performance. He was solid [...] and concluded by launching a big counter attack against the black arts of his unnamed enemies. As an exercise in orchestrated obfuscation, it was top notch" (Telegraph, 6 février 2017).
} 
pas plainte contre Poutou, comme tu l'as susurré lors du débat des 11. Fillon, sors tes preuves ou sors du débat. [Lucifer, 7 avril 201710 h $05 \mathrm{~min}$; http://ripostelaique.com/cabinet-noir-fillon-menace-detout-dire.html]

Ou plus ramassé :

Question bête : pourquoi ne lâche-t-il pas tout de suite ces infos ? Peut être parce que ceux qui ont balancé ont dit la vérité ? [Jarno , 7 avril 201717 h 43 min; http://ripostelaique.com/cabinet-noir-fillonmenace-de-tout-dire.html]

Attaque, tentative de contre-attaque et ultime riposte : en salissant les autres, loin de se laver de tout soupçon, François Fillon se salissait lui-même. Mal engagée, la stratégie 'attacking the accuser' ne réparait pas son image, mais la fragilisait plus encore en se retournant contre lui.

\section{Le pathos affaiblit le logos}

(9) Devant 15000 témoins je veux dire à Penelope que je l'aime. (F. Fillon, 29 janvier 2017, meeting de La Villette)

(10) Mon épouse est remarquable, elle est exceptionnelle, vous n'imaginez pa1s à quel point elle souffre qu'on puisse penser qu'elle n'a pas respecté les règles. Je la défendrai, je l'aime et je la protégerai et je dis à tous ceux qui voudront s'en prendre à elle qu'ils me trouveront en face. (F. Fillon, 27 janvier 2017, interview télévisée, TF1)

Dans ces extraits, c'est d'abord le déplacement du focus du discours qu'opère François Fillon qui apparaît habile, comme pour détourner les feux de l'accusation loin de sa propre personne: 'détournement' ou 'diversion' pourrait-on dire. A l'écouter en effet ce ne sont plus lui et ses actions passées qui sont au cœur des reproches de la justice ou de l'opinion publique, mais sa femme qu'il convient dès lors de défendre. Ici d'accusé, il devient avocat et le protecteur de son épouse dans une posture virile sans doute valorisable.

Mais c'est l'utilisation du pathos à un niveau rarement atteint que nous voulons souligner. La déclaration d'amour ici exhibée " devant 15.000 personnes » (" je l'aime ») est en effet rare en politique et fait écho à une série de choix lexicaux pathémiques [Bonhomme 2014 (2005)] remarquables, dans le contexte précis des affaires et de la restauration d'image entreprise.

L'étude systématique de la campagne 2017 que nous avons entreprise ailleurs [Mayaffre et al. 2017] montre en effet combien, chez François Fillon, aux ci-devant mots de l'amour répondent ceux de la haine de l'autre: " assassinat politique " (F. Fillon, $1^{\mathrm{er}}$ mars 2017, conférence de presse), "lynchage médiatique »(F. Fillon, 6 février 2017, conférence de 
presse), "lyncher, assassiner politiquement, comme vous l'avez fait... " (idem), "je ne pardonnerai jamais à ceux qui ont voulu nous jeter aux loups» (F. Fillon, 29 janvier 2017, meeting de La Villette), " on cherche à me casser " (idem), " ce n'est pas moi seulement qu'on assassine, c'est l'élection présidentielle. [...] " (F. Fillon, $1^{\mathrm{er}}$ mars 2017, conférence de presse), etc. Tant est si bien qu'une chroniqueuse excédée que François Fillon puisse ainsi se comparer implicitement au sort dramatique d'un Jaurès, d'un Kennedy ou d'un Rabin, et alors que le candidat mobilisait le souvenir de Pierre Bérégovoy mort tragiquement, s'emporte en direct à la télévision : «Est-ce que vous nous faites un chantage au suicide ? ! " (C. Angot, 23 mars 2017, interview télévisée de F. Fillon, France 2).

Cette stratégie rhétorique du pathos, à l'amour à la mort, était-elle susceptible d'être gagnante et de réparer l'image d'un candidat dont l'éthos serait ressorti plus sensible, plus humain, plus sympathique au sens propre comme étymologique ? Sans doute... si elle n'était entrée en contradiction directe avec une argumentation du logos, froide, méthodique que le candidat utilise très majoritairement dans la campagne [Mayaffre et al. 2017] et dont il avait fait montre tout le long de sa carrière politique, notamment en tant que Premier ministre de la France austère et rigoureux, sans effusion de sentiments ni débordement passionnel. Car que cherche à faire François Fillon, finalement, pour se réhabiliter ? A expliquer ou à émouvoir ? A s'innocenter par la raison ou à se faire pardonner par les cœurs ?

Le dilemme, pour ne pas dire la contradiction rhétorique, peut être illustré dans la séquence par l'appel froid et raisonnable que François Fillon fait d'abord à la justice devant laquelle il dit vouloir s'expliquer puis, face aux premières décisions défavorables de la justice, par l'appel chaud et passionné au peuple auquel il demande non sans accent populiste de trancher.

Le 25 janvier en effet, le candidat fait savoir par communiqué qu'il demande à être reçu par le Parquet "dans les plus brefs délais " afin de "rétablir la vérité » et de faire taire " cette campagne de calomnie » (Figure 1). 


\section{François Fillon}

" J'ai appris par la presse l'ouverture d'une enquête préliminaire du Parquet national financier.

Cette décision particulièrement rapide permettra de faire taire cette campagne de calomnie et de mettre un terme à ces accusations dénuées de tout fondement.

Je souhaite, pour rétablir la vérité, être reçu au Parquet national financier dans les plus brefs délais.

\section{[...]}

François Fillon

Figure 1. Communiqué de F. Fillon relayé par son compte Twitter le 25 janvier 2017

Seulement, 15 jours après, le 9 février, ses avocats tentent en vain une manœuvre et réclament le dessaisissement dudit Parquet en le jugeant illégitime : le candidat d'accuser désormais la justice de trop de célérité et de partialité. Et lorsque qu'il apprend sa mise en examen, et après avoir prononcé ces mots "d'assassinat politique ", de s'en tourner vers le peuple, contre la justice :

(12) C'est au peuple français que je m'en remets, parce que seul le suffrage, et non une procédure menée à charge, peut décider qui sera le prochain président. (F. Fillon, 6 février 2017, conférence de presse)

Dans une partition devenue indéchiffrable, François Fillon semble ainsi avoir essayé d'abord de jouer la justice contre l'opinion publique (la presse); puis l'opinion publique (le peuple) contre la justice ; ou, dans une cacophonie rhétorique, d'avoir joué la raison judiciaire contre le sentiment politique, puis le sentiment politique contre la raison judiciaire.

\section{Provocation /'provocation'}

(13) Il est là, pour moi, le devoir d'exemplarité du président.

Un président exemplaire, ce n'est pas un homme qui s'expose chaque jour au voyeurisme public dans l'espoir de séduire ou de se faire pardonner d'exercer le pouvoir.

Un président exemplaire, c'est un homme qui propose au peuple ce qu'il croit juste, et lorsqu' il est investi met tout en œuvre pour réaliser le projet sur lequel il a été élu. 
Un président exemplaire, c'est un président qui ne troque pas le destin de la nation pour un peu de popularité éphémère.

Un président exemplaire, c'est un président qui n'a pas un regard pour les manœuvres d'appareil, ce que le général de Gaulle appelait les poisons et les délices du système.

Un président exemplaire, c'est un président que rien ne peut intimider, ni la calomnie, ni les pressions, ni même l'incompréhension, parce qu'il est devenu, après l'élection, comptable des espoirs de tout un peuple. (F. Fillon, 9 avril 2017, meeting de Paris)

L'anaphore rhétorique fut la figure rhétorique gagnante en 2007 pour Nicolas Sarkozy qui en utilisa plusieurs milliers dans un discours sans comparaison historique en la matière (Mayaffre 2015), comme elle le fut plus ponctuellement pour François Hollande en 2012 lors du débat télévisé de l'entre-deux-tours. Suivant ses devanciers, François Fillon tente ainsi son va-tout rhétorique à quelques jours du scrutin. Et ici, l'anaphore est mobilisée par François Fillon pour ce que l'on qualifiera d'abord en français, dans une acception naturelle, de provocation puis en anglais, dans l'outillage conceptuel de William Benoit, de 'provocation'10.

Clairement polémique par la mise en exergue initiale de la subjectivité du locuteur (" pour moi ») et plus encore par la tournure négative (Nølke 1992) de la première formulation ( «... un président exemplaire ce n'est pas un homme...»), François Fillon utilise à nouveau la contre-offensive jusqu'à la provocation. II reprend, il répète, il martèle - c'est le principe de l'anaphore - ainsi sans tabou le devoir d'exemplarité qu'on lui reprochait précisément de ne pas tenir, et donne une contre-définition de l'exemplarité susceptible d'innocenter son comportement et d'accabler ses adversaires. D'un point de vue quantitatif, c'est bien François Fillon qui, à l'occasion de cette fin de campagne, se met à sur-utiliser face à ses concurrents, dans un retournement calculé de la charge lexicale, les deux mots « exemplaire » et « exemplarité » (Figure 2).

\footnotetext{
${ }^{10}$ Très préparé, François Fillon avait déjà rodé l'anaphore sur l'exemplarité quelques jours auparavant dans I'ultime débat télévisé : (14) « Qu'est-ce que c'est qu'un Président exemplaire ? C'est d'abord un Président qui dit la vérité aux Français [...]. Un Président exemplaire, c'est un Président qui met en œuvre les engagements qu'il a pris devant le peuple [...]. Un Président exemplaire, c'est un Président qui respecte son Premier ministre [...]. Un Président exemplaire, c'est un Président qui ne se sert pas des moyens de l'État pour affaiblir ses adversaires. Et un Président exemplaire enfin, c'est un Président qui ne confie pas à des journalistes des secrets défense. Et pour finir, un Président exemplaire, c'est un Président qui au bout de 5 ans, peut dire qu'il a amélioré la situation et la vie des Français. ॥ (F. Fillon, 4 avril 2017, débat télévisé ; BFM-TV)
} 


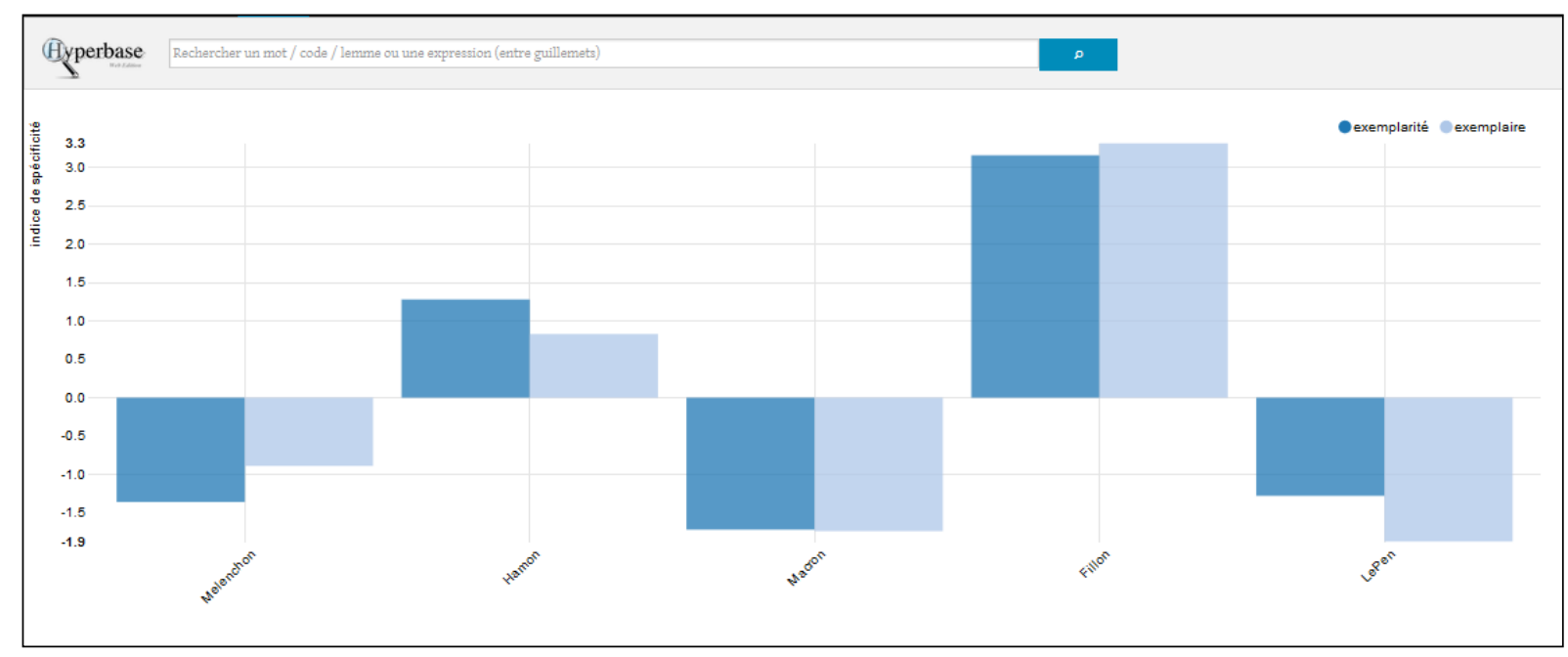

Figure 2. Utilisation de « exemplarité » et « exemplaire » dans la campagne 2017 (sortie Hyperbase Web)

Dans l'extrait 13, poussant le dialogisme inter-discursif polémique et provocateur, il convoque sans complexe à nouveau de Gaulle - celui-là même qu'il avait convoqué pour dénoncer la mise en examen de Nicolas Sarkozy et qu'on lui rappelait depuis sans relâche pour l'inciter à tenir sa promesse de mise en retrait.

Bref, comme indiqué, « rien » ne semble désormais plus pouvoir « intimider » le locuteur. Et derrière ce " rien ", l'auditoire comprend que François Fillon prétend s'affranchir y compris des décisions de justice. Dans un tout pour le tout discursif et politique, François Fillon cherche-t-il ici encore à réparer son image ? Ou espère-t-il finalement se faire élire malgré les handicaps de notoriété dont il commençait à pâtir? Les indicateurs sondagiers devenaient certes difficiles mais n'étaient pas désespérés: avec une barre de qualification pour le second tour pressentie autour de $20 \%$ des votants, François Fillon pouvait encore rallier l'équivalent de 7 ou 8 millions de partisans irrésolus, passer le premier tour et l'emporter au second aussi bien face à Marine Le Pen, Jean-Luc Mélenchon qu'Emmanuel Macron.

Si l'on peut parler avec Benoit également de 'provocation' dans une stratégie d'evading responsibility', c'est que les actes de François Fillon visés par la justice semblent en tout cas dans les extraits 13 et 14 être relativisés par le candidat à la lumière du supposé comportement passé de François Hollande ou du supposé comportement futur d'un Emmanuel Macron. François Fillon a fauté certes, mais n'est-ce pas, au fond, dans une forme de réponse à des fautes plus graves encore pour la France de ses adversaires ? L'argument rhétorique est fragile et ici encore François Fillon semble se battre contre un fantôme (Hollande) ou contre une promesse (Macron) qui ne pouvaient faire oublier l'épaisseur du ici et maintenant du candidat dans une campagne électorale immédiate et sur-médiatisée.

\section{Conclusion}

"Mon image a été abîmée » (F. Fillon, 6 avril 2017, interview radio, France Inter) : le constat a été lucide mais l'analyse aura été trop courte. L'infélicité de la réparation d'image que 
François Fillon tenta s'explique par l'incapacité du candidat, ou son refus, d'identifier ce qu'on pouvait lui reprocher fondamentalement et, en conséquence, l'usage désordonné de stratégies rhétoriques contradictoires à force d'être plurielles (tableau 1). Concédons également, loin des mots et des discours, que les faits, du moins tels qu'ils s'accumulaient dans la presse, puis furent pointés par les réquisitoires de la justice, étaient sans doute trop lourds pour que le locuteur puisse les contrer par la seule force du discours et la magie de la rhétorique.

Tableau 1. Conditions d'infélicité de la restauration d'image de F. Fillon

\begin{tabular}{|c|c|c|c|c|}
\hline Discours & Stratégie argumentative & & Condition d'infélicité & Discours \\
\hline $\begin{array}{l}\text { je présente mes } \\
\text { excuses... }\end{array}$ & 'Mortification' & $\mathrm{C}$ & 'Denial' & $\begin{array}{l}\text {... mais je n'ai pas commis } \\
\text { de faute }\end{array}$ \\
\hline $\begin{array}{l}\text { j'ai rendu les } \\
\text { costumes... }\end{array}$ & 'Compensation' & $\mathrm{O}$ & 'Minimization' & $\begin{array}{l}\ldots \text { mais les posséder n'avait } \\
\text { rien de grave }\end{array}$ \\
\hline $\begin{array}{l}\text { il existe un complot } \\
\text { de la presse et de la } \\
\text { justice contre moi... }\end{array}$ & $\begin{array}{l}\text { 'Attacking accuser', } \\
\text { 'Victimisation' }\end{array}$ & $\begin{array}{l}\mathrm{N} \\
\mathrm{T}\end{array}$ & $\begin{array}{l}\text { Paranoïa, théorie du } \\
\text { complot }\end{array}$ & $\begin{array}{l}\text {...mais je vous en donnerai } \\
\text { la preuve seulement } \\
\text { ultérieurement }\end{array}$ \\
\hline $\begin{array}{l}\text { je l'aime... on } \\
\text { m'assassine... }\end{array}$ & Pathos & $\mathrm{R}$ & Logos & $\begin{array}{l}\text {... seule la France et sa dette } \\
\text { méritent notre attention }\end{array}$ \\
\hline $\begin{array}{lr}\text { Hollande } & \text { (ou } \\
\text { Macron) sont } \quad \text { les } \\
\text { vrais coupables... }\end{array}$ & $\begin{array}{l}\text { 'Provocation', } \\
\text { 'Attacking accuser' }\end{array}$ & $\mathrm{D}$ & Provocation & $\begin{array}{l}\text {... moi, malgré les charges } \\
\text { qui pèsent contre moi, je suis } \\
\text { « exemplaire » }\end{array}$ \\
\hline $\begin{array}{l}\text { je veux servir mon } \\
\text { pays... }\end{array}$ & Désintéressement & $\begin{array}{l}\mathrm{I} \\
\mathrm{C}\end{array}$ & Intéressement & $\begin{array}{l}\text {...j'ai versé } 680.380,78 \text { euros } \\
\text { nets à ma femme, j'ai } \\
\text { employé mes enfants }\end{array}$ \\
\hline $\begin{array}{l}\text { mis en examen, je } \\
\text { me retirerai... }\end{array}$ & Engagement & $\mathrm{T}$ & Insincérité & $\begin{array}{l}\ldots \text { ma mise en examen ne me } \\
\text { fera pas renoncer }\end{array}$ \\
\hline $\begin{array}{l}\text { ma femme était mon } \\
\text { attachée } \\
\text { parlementaire... }\end{array}$ & Vérité ou mensonge? & $\begin{array}{l}\mathrm{O} \\
\mathrm{N}\end{array}$ & Mensonge ou vérité ? & $\begin{array}{l}\text { I have never been actually his } \\
\text { assistant or anything like } \\
\text { that }^{11}\end{array}$ \\
\hline
\end{tabular}

C'est François Bayrou, un ennemi politique intime, qui exprime le sentiment partagé : François Fillon serait sous l'influence " des puissances d'argent » (F. Bayrou, 8 février 2017, interview télévisée, France 2). Et Marine Le Pen, une concurrente dans la course à l'Elysée, de conclure plus directement que François Fillon était « un homme qui aimait l'argent [...] un homme d'argent ». (M. Le Pen, 14 mars 2017, interview télévisée, RFI-France24). Pourtant François Fillon ne cherchera jamais à contrer l'image de l'homme intéressé qui était dressée de lui, allant le plus souvent sur le terrain de la stricte légalité des faits révélés (quand bien même la justice semblait soupçonner quelques irrégularités) ou sur des terrains plus glissants encore comme par exemple celui de la misogynie des attaques contre son épouse (quand bien même il prenait lui-même des accents patriarcaux en disant vouloir " protéger " sa femme). Et quand il accepta de parler d'argent, sa communication fut pour le moins

\footnotetext{
${ }^{11}$ Extrait d'une interview de Pénélope Fillon diffusée par France 2 le 2 février 2017.
} 
maladroite. Calculette en main et face caméra, il indiquait avoir seulement versé à son épouse la moyenne mensuelle de 3677 euros net pendant quinze ans (F. Fillon, 6 février 2017, conférence de presse). La somme globale en jeu et le salaire mensuel semblaient anodins pour le candidat de droite, là où la rémunération médiane en France durant la période n'atteignait pas 1500 euros. De même, s'il dit avoir rendu les costumes de luxe, il ne justifia jamais leurs coûts équivalents, pour chacun des modèles Arnys visés, à quatre ou cinq smics. Dans la campagne médiatique et judiciaire tenace et tous azimuts dont François Fillon a été le centre pendant 3 mois, la presse épargna à l'homme le soupçon de luxure, mais il semblait désormais établi, sans réel démenti du candidat, que François Fillon était un homme de lucre.

Surtout, et nous touchons au point nodal et, hélas pour le candidat, le point aporétique de la situation, François Fillon ne mesura jamais que c'était sa parole elle-même qui était attaquée et remise en cause, à partir de sa mise en examen et du maintien, malgré sa promesse, de sa candidature. C'est-à-dire que c'était l'outil même ou la condition même de la réparation d'image - le discours - qui se trouvait endommagé par les palinodies du candidat que relayait la presse. En résumé : François Fillon perdait de sa notoriété parce qu'il était accusé de dire des mots-mensonges et de formuler de fausses-promesses et, dès lors, comment pouvait-il encore parler et convaincre l'opinion publique, par ce langage discrédité, qu'il était intègre et disait la vérité ? Dans le cadre logocratique d'une campagne électorale durant laquelle l'action politique se résume à parler, et à parler-vrai, l'image de François Fillon était attaquée précisément sur l'insincérité voire l'illégitimité de sa parole : c'était la probité du locuteur autant que celle de l'homme politique qui était discutée ; c'était le locuteur autant que I'homme politique qui était disqualifié. Dans ces conditions, loin de restaurer l'image, chaque mot prononcé avait pour conséquence d'aggraver le mal et chaque discours concourait au discrédit; un anti-acte de langage ou un contre-performatif d'une certaine manière qui défaisait ce qu'il prétendait construire ou restaurer. Image brisée et (parce que) voix cassée : comment réparer l'irréparable?

\section{Références}

Amossy R. (2010), La présentation de soi. Ethos et identité verbale, Paris, Presses universitaires de France.

Austin J. L. (1962), How to do things with Words: The William James Lectures delivered at Harvard University in 1955, Oxford, Ed. Urmson.

Benoit W. L. (1995), Accounts, Excuses, and Apologies: A Theory of Image Restoration Strategies, New York, State University of New York Press.

Benoit W. L. (1997), « Hugh Grant's image restoration discourse: An actor apologizes », Communication Quarterly 45(3), p. 251-267. 
Benoit W. L. \& Hanczor R. S. (1994), « The Tonya Harding Controversy: An analysis of image restoration strategies », Communication Quarterly 42(4), p. 416-433.

Benoit W. L., \& Brinson, S. L. (1999), «Queen Elizabeth's image repair discourse: Insensitive royal or compassionate queen? », Public Relations Review 25(2), p. 145-156.

Benoit W. L., \& Brinson, S. L. (1994), « AT\&T: “Apologies are not enough.” » Communication Quarterly 42(1), p. 75-88.

Bonhomme M. (2014 [2005]), Pragmatique des figures du discours, Paris, H. Champion.

Brinson S. L. \& Benoit W.L. (1996), « Dow Corning's image repair strategies in the breast implant crisis », Communication Quarterly 44(1), p. 29-41.

Coombs W. T. (2006), « Crisis Management: A communicative approach » dans Botan C. H. \& Hazleton V. (eds), Public Relations Theory II, Mahwah, NJ, Lawrence Erlbaum Associates, p. 171-197.

Mayaffre D. (2015), « L'anaphore rhétorique. Figure des figures du discours électoral de Sarkozy ", Pratiques. Linguistique, Littérature, Didactique p. 165-166, [http://pratiques.revues.org/2418\#tocto2n1].

Mayaffre D. et al. (2017), « Les mots des candidats, de "allons" à "vertu" », dans Perrineau P. (dir.), Le vote disruptif. Les élections présidentielles et législatives de 2017, Paris, Presses SciencesPo, p. 129-152 [hal-01635941].

Nølke H. (1992), "Ne... pas : négation descriptive ou polémique ? ", Langue française 94, p. 65-66.

Searle J. (1969) Speech Acts: An Essay in the Philosophy of Language, Cambridge, Cambridge Press University. 